\title{
Chi 3 dispersion in planar tantalum pentoxide waveguides in the telecommunications window
}

\author{
Ruiqi Y. Chen, ${ }^{1, *}$ Martin D. B. Charlton, ${ }^{1}$ and Pavlos G. Lagoudakis ${ }^{2}$ \\ ${ }^{1}$ School of Electronics and Computer Science, University of Southampton, Southampton, SO17 1BJ, UK \\ ${ }^{2}$ School of Physics and Astronomy, University of Southampton, Southampton, SO17 1BJ, UK \\ *Corresponding author: rickeychen@gmail.com
}

Received December 16, 2008; revised March 5, 2009; accepted March 6, 2009; posted March 10, 2009 (Doc. ID 105299); published March 31, 2009

We report on the dispersion of the third-order nonlinear susceptibility ( $\chi^{(3)}$ or "Chi 3 ") in planar $\mathrm{Ta}_{2} \mathrm{O}_{5}$ waveguides in the telecommunications spectral window. We utilize the observation of third-harmonic generation under ultrashort pulsed excitation as a reference-free characterization method of $\chi^{(3)}$ and obtain a large nonlinear coefficient, $2 \times 10^{-13} \mathrm{esu}$, at $1550 \mathrm{~nm}$. Our observation of efficient third-harmonic generation in $\mathrm{Ta}_{2} \mathrm{O}_{5}$ waveguides in the telecoms window reveals the potential of this material system in high-speed integrated nonlinear optical switches. (C) 2009 Optical Society of America

OCIS codes: $160.4330,230.7390,190.2620,190.4390$.

Fast low-power all-optical switches are a key component for high-bit-rate communication systems. Silica optical fibers are currently used owing to their low loss. However, the small nonlinear refractive index of silica [1-3] requires high switching powers and very long interaction lengths. To achieve compact switching devices, a large nonlinear refractive index $\left(n_{2}\right)$ is required. Although many materials with a higher nonlinear refractive index than silica have been reported [2,4-6], there is a particular interest in those compatible with planar processing technologies, with minimal losses and absorption at a wide range of operating wavelengths, and good thermal and mechanical robustness. Silicon provides one potential candidate $[7,8]$ but exhibits strong free carrier and twophoton absorption limiting device performance. Chalcogenide (e.g., $\mathrm{As}_{2} \mathrm{~S}_{3}$ ) glass is an alternative that does not exhibit two-photon absorption [9-12]; however, it has a relatively low damage threshold (9 GW/ $\mathrm{cm}^{2}$ [13]) compared to $\mathrm{Ta}_{2} \mathrm{O}_{5}$ [14]. Both of the above two examples have large normal material dispersion, which can be detrimental to wavelength division multiplexing applications but can be compensated by adjusting the waveguide geometry dimensions [7-12]. Here we investigate $\mathrm{Ta}_{2} \mathrm{O}_{5}$ rib planar waveguides as an alternative for compact optical devices with a small effective area that could enable the exploitation of nonlinear effects at low light intensity in the telecoms window. $\mathrm{Ta}_{2} \mathrm{O}_{5}$ has been widely used as a high dielectric gate material for microelectronic devices, and multilayer dielectric mirrors, and more recently it was shown to possess a high nonlinear refractive index at $800 \mathrm{~nm}[1,14]$.

Silicon dioxide $\left(\mathrm{SiO}_{2}\right)$ clad $\mathrm{Ta}_{2} \mathrm{O}_{5}$ planar rib waveguides are grown following the methodology in [15]. We examine the nonlinear optical properties of 5 -mm-long waveguides with $750 \mathrm{~nm}$ core thickness and $2.5 \mu \mathrm{m}$ (sample A) and $18 \mu \mathrm{m}$ (sample B) width. Figure 1 shows a cross-sectional scanning electron microscope view of two waveguides. The samples are optically excited using an optical parametric ampli- fier tunable in the near IR ( $280 \mathrm{fs}$ pulses) with no laser-induced damage to the waveguides.

We characterize the nonlinear optical properties of the rib waveguides by spectrally and spatially resolving the guided optical modes at the end face of the rib structure in both the near-IR and visible spectrum. Figure 1 shows the observed spectral broadening for $8 \mathrm{~nJ}$ pulse energy at $1610 \mathrm{~nm}$. This is primarily attributed to self-phase modulation, in accordance with previous studies at $800 \mathrm{~nm}$ [1]. The dependence of
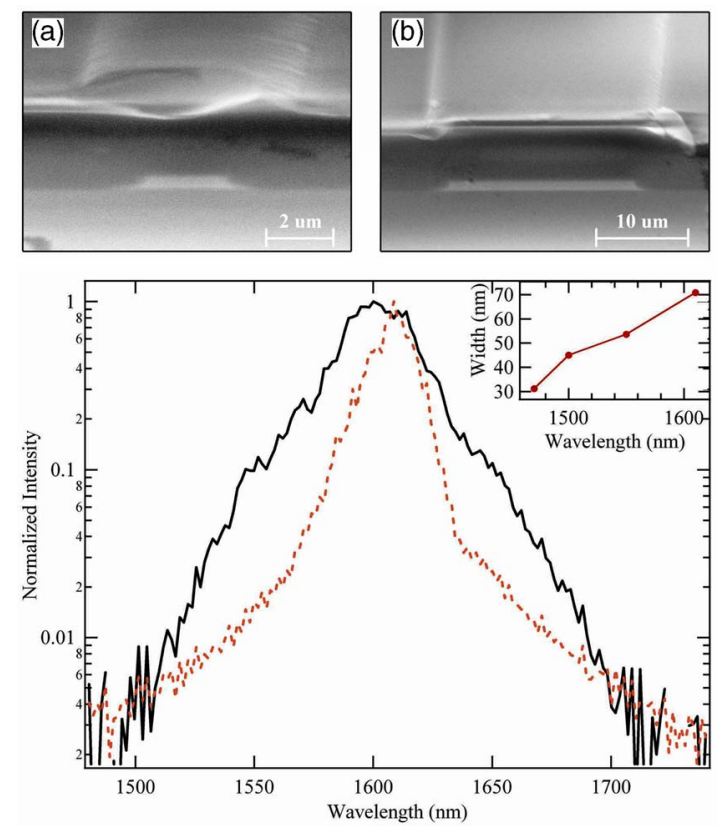

Fig. 1. (Color online) Scanning electron microscope images of the cross-sectional view of the waveguides: (a) sample A (2.5 $\mu \mathrm{m}$ wide), (b) sample B (18 $\mu \mathrm{m}$ wide). Spectral broadening of the pump beam at the end facet of sample A, solid curve. The dotted curve indicates the excitation pump beam with $8 \mathrm{~nJ}$ pulse energy centered at $1610 \mathrm{~nm}$. Inset, dependence of the spectral broadening on excitation wavelength in the telecoms spectral window (constant excitation pulse energy $8 \mathrm{~nJ}$ ). 
the spectral broadening on the excitation wavelength for a constant excitation pulse energy (Fig. 1 inset) suggests a weak dispersion of the nonlinear refractive index $n_{2}$. Although earlier estimates of $n_{2}$ at $800 \mathrm{~nm}$ assumed negligible group velocity dispersion, our observation implies that this must be considered in the telecoms window. The rich structure of the spectrally broadened pump (Fig. 1) further suggests concurrence of other nonlinear processes that hinders a fair estimate of the nonlinear coefficient and its dispersion. Indeed, we clearly observe green light at the end facet of the waveguide, visible to the eye, indicating efficient third-harmonic generation (THG). Interestingly, THG (Fig. 2) is not generated on the fundamental mode but on a higher-order mode that changes with waveguide width.

THG is a purely electronic process spectrally separated from other nonlinear effects. We utilize analysis of the THG signal to obtain $\chi^{(3)}$ dispersion in the
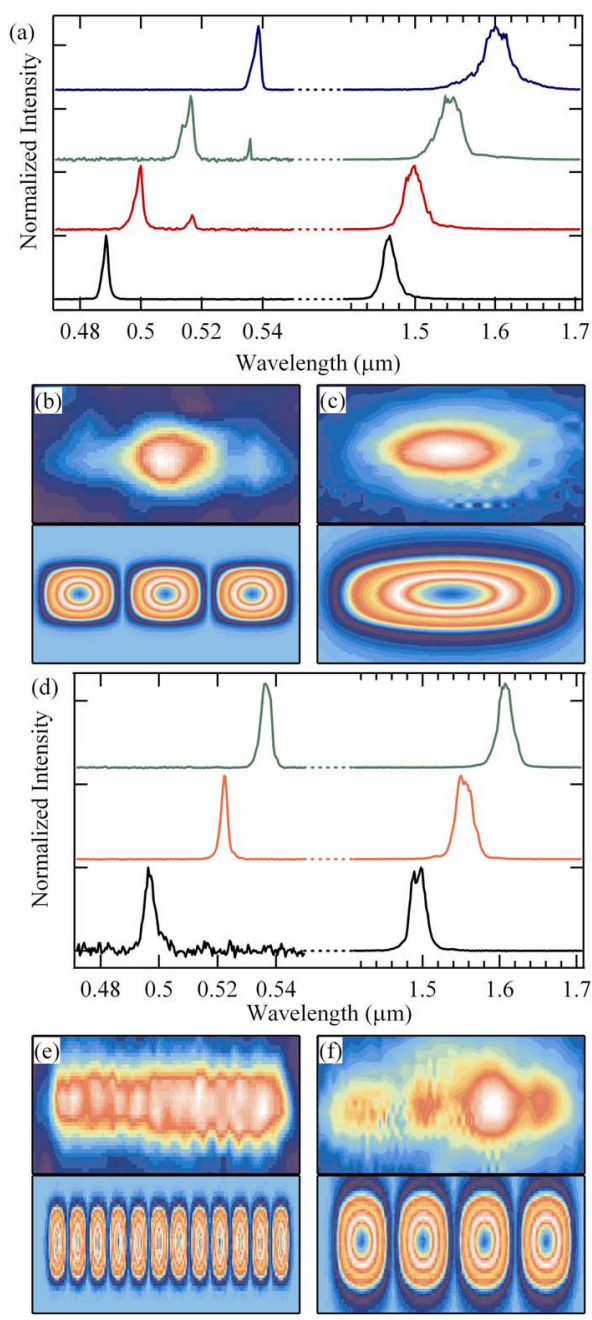

Fig. 2. (Color online) Third-harmonic spectra and corresponding pump spectra over a range of telecoms and IR wavelengths with respective spatial and calculated mode profiles for waveguide samples A and B. (a) Third-harmonic spectra and corresponding pump spectra for sample A. (b) and (c) Spatial mode profiles in the visible and near IR with the respective calculated modes for sample A. (d) Thirdharmonic spectra and corresponding pump spectra for sample B. (e) and (f) Spatial mode profiles in visible and near IR with the respective calculated modes for sample B. telecoms window. Under optimum phase matching conditions,

$$
\left|\chi^{(3)}\right|=\left[I(3 \omega) \frac{\left(c n_{0}\right)^{4} \Delta k^{2} \varepsilon_{0}^{2}}{4 \omega^{2} I(\omega)^{3}}\right]^{1 / 2},
$$

where $\omega=(2 \pi c) / \lambda, \Delta k=k(3 \omega)-3 k(\omega)$, and $I$ is the intensity per unit area. We calculate the effective mode indices and wave vectors $(k)$ for the recorded spatial mode profiles of the THG signal at the corresponding wavelengths by finite-element analysis, taking into account material dispersion $[16,17]$ and with numerical accuracy set to the tenth digit. Figures 3(a) and 3 (b) show the calculated effective index for the THG signal, fundamental, pump beam, and the resulting $\Delta k$. The simulations show that for the THG signal of different modes/waveguides, $\Delta k$ is unchanged. This indicates a self-selective phase matching optimization process inside the waveguides. Experimentally, THG is optimized by fine tuning the pump wavelength to maximize its intensity. Whereas an estimate of $\chi^{(3)}$ dispersion can be obtained by measuring the intensity of the THG signal and of the pump beam coupled in the waveguide, as well as their spatial mode profiles for different pump wavelengths, the indeterminacy of the coherence length in our structures prevents an absolute measure of $\chi^{(3)}$. Hereafter we will obtain a lower estimate of $\chi^{(3)}$ dispersion as a result of possible deviations from optimum phase matching conditions in the process of THG.

To remove ambiguities associated with the efficiency of the input coupling we determine the pump intensity contributing to THG by scaling the measured intensity of the transmitted pump beam at the

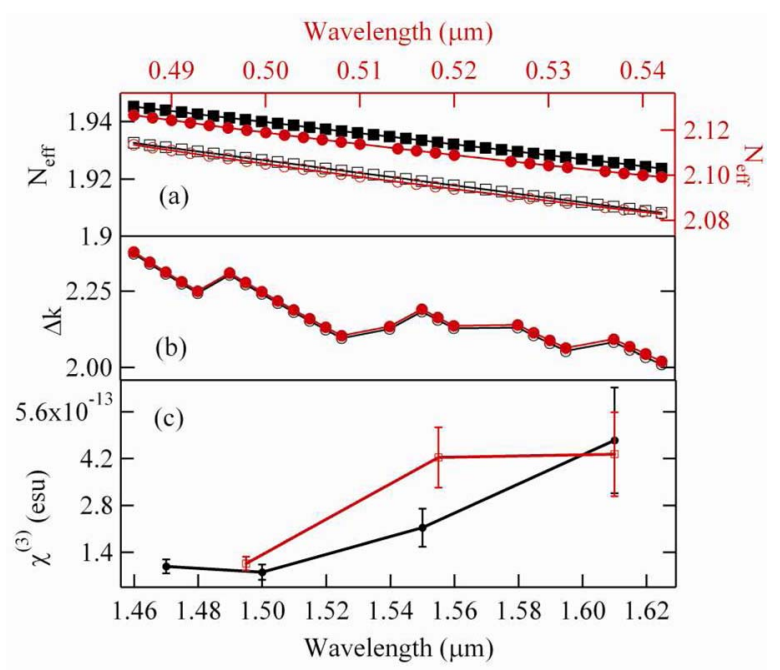

Fig. 3. (Color online) Phase matching and $\chi^{(3)}$ (Chi 3) dispersion in the waveguides. (a) Calculated effective index for the third-harmonic signal with open circles and solid circles for sample A and B, respectively. The fundamental pump beam with open squares and solid squares for sample $\mathrm{A}$ and $\mathrm{B}$, respectively. (b) Resulting $\Delta k$ with open circles for sample A and solid circles for sample B. (c) $\chi^{(3)}$ (Chi 3) dispersion, on wavelength: solid circles for sample A and open squares for sample B. 
Table 1. Calculated Nonlinear Coefficient for Each Pump Wavelength

\begin{tabular}{clc}
\hline Sample & $\lambda_{\mathrm{THG}}(\mathrm{nm})$ & $\chi^{(3)}(\mathrm{esu})$ \\
\hline \multirow{2}{*}{$\mathrm{A}$} & 489 & $(9.76 \pm 2.10) \times 10^{-14}$ \\
& 500 & $(8.02 \pm 2.31) \times 10^{-14}$ \\
& 516 & $(2.13 \pm 5.68) \times 10^{-13}$ \\
& 538 & $(4.75 \pm 1.59) \times 10^{-13}$ \\
$\mathrm{~B}$ & 497 & $(4.23 \pm 0.21) \times 10^{-13}$ \\
& 522 & $(4.33 \pm 1.26) \times 10^{-13}$ \\
& 536 & \\
\hline
\end{tabular}

output of the waveguide. We account for the waveguide losses measured at $-8.5 \mathrm{~dB} / \mathrm{cm}$ using the cutback method on identical waveguide samples from the same wafer. We also correct for the component of the spectral integral that is present in the transmitted pump beam due to self-phase modulation and account for the losses of the recorded light intensities from our optical detection path and detector, thus obtaining $\chi^{(3)}$ values of $\mathrm{Ta}_{2} \mathrm{O}_{5}$ planar waveguides.

Table 1 shows the calculated nonlinear coefficient for the two waveguides in the telecoms window and for constant pump beam intensity of $8 \mathrm{~nJ}$. Figure 3(c) shows $\chi^{(3)}$ dispersion in $\mathrm{Ta}_{2} \mathrm{O}_{5}$, which increases significantly with wavelength. Error bars are calculated from the propagating error in the measurement of the waveguide losses, estimated at $10 \%$ [14]. The average value for $\chi^{(3)}$ in the frequency range of 1470 to $1610 \mathrm{~nm}$ is $(3.14 \pm 1.62) \times 10^{-13} \mathrm{esu}$ in agreement with earlier estimates in the near IR [1]. Thus, the value of the nonlinear susceptibility of $\mathrm{Ta}_{2} \mathrm{O}_{5}$ is estimated to be between the $\mathrm{BK} 7$ silica glasses $\left(\chi^{(3)}\right.$ $\left.\approx 2.0 \times 10^{-14} \mathrm{esu}\right)$ and titanium dioxides $\left(\mathrm{TiO}_{2}\right)\left(\chi^{(3)}\right.$ $\approx 1.5 \times 10^{-12} \mathrm{esu}$ ).

In conclusion, we observe a third-harmonic signal in high-quality $\mathrm{Ta}_{2} \mathrm{O}_{5}$ planar rib waveguides over wavelengths in the telecoms window. We experimentally obtained the nonlinear susceptibility $\chi^{(3)}$ and observed a strong dispersion of $\chi^{(3)}$ in $\mathrm{Ta}_{2} \mathrm{O}_{5}$. With the introduction of photonic crystals further dispersion engineering becomes possible, making $\mathrm{Ta}_{2} \mathrm{O}_{5}$ a suitable base material for on-chip planar laser and signal processing devices such as supercontinuum generation, optical parametric amplifiers, and nonlinear optical switching. The compatibility with silicon processing technologies, the high index, and the large value of $\chi^{(3)}$ that we obtain render $\mathrm{Ta}_{2} \mathrm{O}_{5}$ waveguides a strong candidate, competitive with silicon, chalcogenide, or titanium dioxide $\left(\mathrm{TiO}_{2}\right)$, for alloptical integrated circuits and switching devices.

\section{References}

1. C.-Y. Tai, J. Wilkinson, N. Perney, M. Netti, F. Cataneo, C. Finlayson, and J. Baumberg, Opt. Express 12, 5110 (2004).

2. R. W. Boyd, Nonlinear Optics, 2nd ed. (Academic, 2003).

3. B. P. Nelson, K. J. Blow, P. D. Constantine, N. J. Doran, J. K. Lucek, I. W. Marshall, and K. Smith, Electron. Lett. 27, 704 (1991).

4. A. Samoc, M. Samoc, M. Woodruff, and B. LutherDavies, Opt. Lett. 20, 1241 (1995).

5. T. Hashimoto and T. Yoko, Appl. Opt. 34, 2941 (1995).

6. M. Hemissi and H. Amardjia-Adnani, Dig. J. Nanomater. Biostruct. 2, 299 (2007).

7. J. I. Dadap, N. C. Panoiu, X. Chen, I. W. Hsieh, X. Liu, C.-Y. Chou, E. Dulkeith, S. J. McNab, F. Xia, W. M. J. Green, L. Sekaric, Y. A. Vlasov, and J. R. M. Osgood, Opt. Express 16, 1280 (2008).

8. I. W. Hsieh, X. Chen, J. I. Dadap, N. C. Panoiu, R. M. Osgood, S. J. McNab, and Y. A. Vlasov, Opt. Express 14, 12380 (2006).

9. M. R. Lamont, B. Luther-Davies, D.-Y. Choi, S. Madden, and B. J. Eggleton, Opt. Express 16, 14938 (2008).

10. M. R. Lamont, B. Luther-Davies, D.-Y. Choi, S. Madden, X. Gai, and B. J. Eggleton, Opt. Express 16, 20374 (2008).

11. M. D. Pelusi, V. G. Ta'eed, F. Libin, E. Magi, M. R. E. Lamont, S. Madden, C. Duk-Yong, D. A. P. Bulla, B. Luther-Davies, and B. J. Eggleton, IEEE J. Sel. Top. Quantum Electron. 14, 529 (2008).

12. V. Ta'eed, M. D. Pelusi, B. J. Eggleton, D.-Y. Choi, S. Madden, D. Bulla, and B. Luther-Davies, Opt. Express 15, 15047 (2007).

13. R. Stegeman, G. Stegeman, P. Delfyett, L. Petit, N. Carlie, K. Richardson, and M. Couzi, Opt. Express 14, 11702 (2006).

14. C. M. Netti, M. E. Zoorob, S. W. Roberts, M. D. B. Charlton, G. J. Parker, J. J. Baumberg, J. R. Lincoln, F. Tauser, A. Zach, G. Flinn, and F. Lison, in Commercial and Biomedical Applications of Ultrafast Lasers V (SPIE, 2005), pp. 195-199.

15. J. D. Mills, T. Chaipiboonwong, W. S. Brocklesby, M. D. B. Charlton, M. E. Zoorob, C. Netti, and J. J. Baumberg, Opt. Lett. 31, 2459 (2006).

16. G. E. Jellison and F. A. Modine, Appl. Phys. Lett. 69, 371 (1996).

17. K. Postava, M. Aoyama, T. Yamaguchi, and H. Oda, Appl. Surf. Sci. 175-176, 276 (2001). 